\title{
On Semianalytical Study of Fractional-Order Kawahara Partial Differential Equation with the Homotopy Perturbation Method
}

\author{
Muhammad Sinan $\left(\mathbb{D},{ }^{1,2}\right.$ Kamal Shah $\mathbb{D}^{3},{ }^{3}$ Zareen A. Khan $\mathbb{D}^{4},{ }^{4}$ Qasem Al-Mdallal $\left(\mathbb{D},{ }^{5}\right.$ \\ and Fathalla Rihan $\mathbb{1 D}^{5}$ \\ ${ }^{1}$ School of Mathematical Sciences, University of Electronic Science and Technology of China, Chengdu 611731, China \\ ${ }^{2}$ Department of Mathematics and Statistics, University of Swat, Swat, Khyber Pakhtunkhwa, Pakistan \\ ${ }^{3}$ Department of Mathematics, University of Malakand, Chakdara Dir (L) 18000, Khyber Pakhtunkhwa, Pakistan \\ ${ }^{4}$ College of Science, Mathematical Sciences, Princess Nourah Bint Abdulrahman University, Riyadh, Saudi Arabia \\ ${ }^{5}$ Department of Mathematical Sciences, UAE University, P.O. Box 15551, Al-Ain, UAE
}

Correspondence should be addressed to Fathalla Rihan; frihan@uaeu.ac.ae

Received 7 August 2021; Revised 5 October 2021; Accepted 11 October 2021; Published 3 November 2021

Academic Editor: Ndolane Sene

Copyright (c) 2021 Muhammad Sinan et al. This is an open access article distributed under the Creative Commons Attribution License, which permits unrestricted use, distribution, and reproduction in any medium, provided the original work is properly cited.

\begin{abstract}
In this study, we investigate the semianalytic solution of the fifth-order Kawahara partial differential equation (KPDE) with the approach of fractional-order derivative. We use Caputo-type derivative to investigate the said problem by using the homotopy perturbation method (HPM) for the required solution. We obtain the solution in the form of infinite series. We next triggered different parametric effects (such as $x$, $\mathrm{t}$, and so on) on the structure of the solitary wave propagation, demonstrating that the breadth and amplitude of the solitary wave potential may alter when these parameters are changed. We have demonstrated that He's approach is highly effective and powerful for the solution of such a higher-order nonlinear partial differential equation through our calculations and simulations. We may apply our method to an additional complicated problem, particularly on the applied side, such as astrophysics, plasma physics, and quantum mechanics, to perform complex theoretical computation. Graphical presentation of few terms approximate solutions are given at different fractional orders.
\end{abstract}

\section{Introduction}

PDEs have important applications in physics, engineering, and other applied sciences. They can describe different phenomena and processes of real-world problems. One of the important KPDE arises in the theory of magnetoacoustic and shallow-water waves. Furthermore, it arises in the theory of shallow-water waves with surface tension and magnetoacoustic waves in plasmas. Therefore, several analytical and numerical methods have been established in literature to investigate the prosed problems of PDEs. For instance, [1] authors have used the comparison method for the solution of the famous Kawahara equation. In the same line, a procedure was developed in [2] for the exact solution of the said problem. Also, authors [3] have computed the solution of the Kawahara equation by using symbolic computation. In this study, we apply a semianalytic HPM to solve the fifth-order KPDEs. As in the last several decades' investigation, traveling-waves solutions for nonlinear equations played an important role in the study of the nonlinear physical phenomenon [4]. The mentioned method provides an efficient approach to solve a nonlinear problem. The KPDE was first suggested by Kawahara [5] in 1972. Since these nonlinear equations need to be solved by using some approximate methods, researchers have solved several nonlinear problems by using HPM. This method was first proposed by $\mathrm{He}$ [6] and has been applied in [7] for the solution of differential equations and integral equations in both linear and nonlinear cases. The said method is a combination of topological homotopy and traditional perturbation methods. The advantage of this method is to provide an analytic approximate solution in applied sciences 
with a capacious range, and in this method, a small parameter is not necessary for an equation. This method is also applied to the system of the nonlinear system of equations as in [8] for the analytic approximate solution for the model of rabies transmission dynamics.

Because of the popularity of fractional calculus and applications in many fields of science and engineering [9], fluid mechanics [10], some more frequent applications in a diverse area of science by using fraction calculus have been investigated in $[11,12]$. The mentioned derivative extends order from integer to any real or complex number which provides a detailed explanation to physical problems. Fractional derivatives can produce a complete spectrum of the geometry which includes its integer counterpart as a special case. Motivated from the aforesaid work, we extend the given KPDE [13].

$$
\begin{aligned}
\frac{\partial u}{\partial t}+\rho u \frac{\partial u}{\partial x}+\sigma \frac{\partial^{3} u}{\partial x^{3}}-\gamma \frac{\partial^{5} u}{\partial x^{5}} & =0, \\
u(x, 0) & =g(x),
\end{aligned}
$$

where $g$ is a continuous function, while $\rho, \sigma$, and $\gamma$ are the nonzero arbitrary constants to fractional order as

$$
\begin{aligned}
\frac{\partial^{\alpha} u}{\partial t^{\alpha}}+\rho u \frac{\partial^{\beta} u}{\partial x^{\beta}}+\sigma \frac{\partial^{\kappa} u}{\partial x^{\kappa}}-\gamma \frac{\partial^{\delta} u}{\partial x^{\delta}} & =0, \quad 0<\alpha \leq 1, \\
u(x, 0) & =g(x),
\end{aligned}
$$

while $0<\beta \leq 1,2<\kappa \leq 3,4<\delta \leq 5$, and $g: R \longrightarrow R$ is continuous function.

For the demonstration of our problem, we testified the example given by Albert [14] as

$$
\frac{\partial^{\alpha} u}{\partial t^{\alpha}}+\rho u \frac{\partial^{\beta} u}{\partial x^{\beta}}+\sigma \frac{\partial^{\kappa} u}{\partial x^{\kappa}}-\gamma \frac{\partial^{\delta} u}{\partial x^{\delta}}=0,
$$

with the initial condition

$$
u(x, 0)=\frac{105}{169} \operatorname{sech}^{4}\left(\frac{x}{2 \sqrt{13}}\right),
$$

by using fractional derivative in Caputo sense. We also present the solutions graphically and then, at the end, provide conclusion and discussion.

\section{Preliminaries and Notations}

Here, we recall some preliminaries and notations from [15].

Definition 1. Caputo fractional-order derivative of a function $\phi$ on the interval $[0, \infty)$ is defined as

$$
{ }^{c} D_{0+}^{\alpha} \phi(t)=\frac{1}{\Gamma(n-\alpha)} \int_{0}^{t}(t-s)^{n-\alpha-1} \frac{\mathrm{d}^{n}}{\mathrm{~d} s^{n}} \phi(s) \mathrm{d} s,
$$

where $n=[\alpha]+1$ and $[\alpha]$ represent the integral part of $\alpha$.

Definition 2. If $\alpha>0$, the Caputo fractional integral is defined as

$$
I^{\alpha} \phi(t)=\frac{1}{\Gamma(\alpha)} \int_{0}^{t}(t-s)^{\alpha-1} \phi(s) \mathrm{d} s,
$$

where $\alpha \in(0, \infty)$.

Lemma 1. The following result holds:

$$
I^{n}\left[C D_{0+}^{n} \phi(t)\right]=\phi(t)+a_{0}+a_{1} t+a_{2} t^{2}+\cdots+a_{n-1} t^{n-1},
$$

for arbitrary $a_{i} \in R, i=0,1,2,3, \ldots, n-1$, where $n=[\eta]+1$ and $[\eta]$ represent the integral part of $\eta$.

\section{A General Algorithm about HPM}

Consider a general type problem given by

$$
A(\mu)-f(r)=0, \quad r \in \Omega,
$$

with boundary condition as

$$
\beta\left(\mu, \frac{\partial \mu}{\partial n}\right)=0, r \in \Gamma,
$$

where $A$ is a general differential operator, $\beta$ is a boundary operator, $f(r)$ is a known analytic function, and $\Gamma$ is the boundary of the domain $\Omega$. The operator $A$ is divided into linear part $L$ and nonlinear part $N$. Therefore, (9) can be written as

$$
L(\mu)+N(u)-f(r)=0 .
$$

By HPM, we can construct a homotopy as

$$
v(r, p): \Omega \times[0,1] \longrightarrow R,
$$

satisfying

$$
H(v, p)=(1-p)[L(v)-L(\mu)]+p[A(v-f(r))]=0,
$$

which is also equivalent to

$$
H(v, p)=L(v)-L\left(\mu_{0}\right)+p L\left(v_{0}\right)+p[N(v)-f(r)]=0,
$$

where $p \in[0 ; 1]$ is an embedding parameter, and $\mu_{0}$ is the initial approximation of the given equation that satisfies the boundary conditions; we have

$$
\begin{aligned}
& H(v, 0)=L(v)-L\left(\mu_{0}\right)=0, \\
& H(v, 1)=A(v)-f(r)=0 .
\end{aligned}
$$

Keeping these points, we construct the required solution to equation (11) as

$$
v=v_{0}+p^{1} v_{1}+p^{2} v_{2}+p^{3} v_{3}+\cdots .
$$

Furthermore, by taking limit as $p \longrightarrow 1$ in the approximation equation (16), one has

$$
\lim _{p \longrightarrow 1} v=\lim _{p \longrightarrow 1} v_{0}+p^{1} v_{1}+p^{2} v_{2}+p^{3} v_{3}+\cdots,
$$

which yields 


$$
v=v_{0}+v_{1}+v_{2}+v_{3}+\cdots .
$$

Equation (18) represents the semianalytic solution of the problem equation (9).

\section{Approximate Solution to Considered Problem}

Here, in view of HPM as discussed in previous section, we proceed as

$$
H(u, p)=\frac{\partial^{\alpha} u}{\partial t^{\alpha}}-\frac{\partial^{\alpha}}{\partial t^{\alpha}} g(x)+p \frac{\partial^{\alpha}}{\partial t^{\alpha}} g(x)+p\left[\alpha u \frac{\partial^{\beta} u}{\partial x^{\beta}}+\sigma \frac{\partial^{\kappa} u}{\partial x^{\kappa}}-\gamma \frac{\partial^{\delta} u}{\partial x^{\delta}}\right]=0
$$

We assume the solution of equation (2) as follows:

$$
u(x, t)=\sum_{i=0}^{\infty} p^{i} u_{i}(x, t)
$$

Now, using equation (20) in (19) and comparing the coefficients of $p^{i}$, for $i=0,1,2,3, \ldots$, we have

$$
\begin{aligned}
& p^{0}:=\frac{\partial^{\alpha} u_{0}}{\partial t^{\alpha}}-\frac{\partial^{\alpha}}{\partial t^{\alpha}} g(x)=0 \\
& p^{1}:=\frac{\partial^{\alpha} u_{1}}{\partial t^{\alpha}}+\rho u_{0} \frac{\partial^{\beta}}{\partial x^{\beta}} u_{0}+\sigma \frac{\partial^{\kappa}}{\partial x^{\kappa}} u_{0}-\gamma \frac{\partial^{\delta}}{\partial x^{\delta}} u_{0}=0 \\
& p^{2}:=\frac{\partial^{\alpha} u_{2}}{\partial t^{\alpha}}+\rho u_{1} \frac{\partial^{\beta}}{\partial x^{\beta}} u_{1}+\sigma \frac{\partial^{\kappa}}{\partial x^{\kappa}} u_{1}-\gamma \frac{\partial^{\delta}}{\partial x^{\delta}} u_{1}=0 \\
& p^{3}:=\frac{\partial^{\alpha} u_{3}}{\partial t^{\alpha}}+\rho u_{2} \frac{\partial^{\beta}}{\partial x^{\beta}} u_{2}+\sigma \frac{\partial^{\kappa}}{\partial x^{\kappa}} u_{2}-\gamma \frac{\partial^{\delta}}{\partial x^{\delta}} u_{2}=0 \\
& p^{4}:=\frac{\partial^{\alpha} u_{4}}{\partial t^{\alpha}}+\rho u_{3} \frac{\partial^{\beta}}{\partial x^{\beta}} u_{3}+\sigma \frac{\partial^{\kappa}}{\partial x^{\kappa}} u_{3}-\gamma \frac{\partial^{\delta}}{\partial x^{\delta}} u_{3}=0,
\end{aligned}
$$

$$
\begin{aligned}
\frac{\partial^{\alpha} u_{2}}{\partial t^{\alpha}} & =\frac{\gamma t^{\alpha}}{\Gamma(\alpha+1)} \frac{\partial^{\delta}}{\partial x^{\delta}} \xi-\frac{\rho \xi t^{2 \alpha}}{\Gamma(\alpha+1)^{2}} \frac{\partial^{\beta}}{\partial x^{\beta}} \xi-\frac{\sigma t^{\alpha}}{\Gamma(\alpha+1)} \frac{\partial^{\kappa}}{\partial x^{\kappa}} \xi, \\
u_{2} & =\frac{\gamma t^{2 \alpha}}{\Gamma(\alpha+2)} \frac{\partial^{\delta}}{\partial x^{\delta}} \xi-\frac{\rho \xi t^{3 \alpha}}{\Gamma(\alpha+2)^{2} \Gamma(\alpha+3)} \frac{\partial^{\beta}}{\partial x^{\beta}} \xi-\frac{\sigma t^{2 \alpha}}{\Gamma(\alpha+2)} \frac{\partial^{\kappa}}{\partial x^{\kappa}} \xi .
\end{aligned}
$$

Where 


$$
\xi=\gamma \frac{\partial^{\delta}}{\partial x^{\delta}} u_{0}-\rho u_{0} \frac{\partial^{\beta}}{\partial x^{\beta}} u_{0}-\sigma \frac{\partial^{\kappa}}{\partial x^{\kappa}} u_{0}
$$

In the same way, one has

Third-order problem

$$
\begin{aligned}
u_{3}= & \gamma \frac{\partial^{\delta}}{\partial x^{\delta}}\left(\frac{\gamma t^{3 \alpha}}{\Gamma(\alpha+3)} \frac{\partial^{\delta}}{\partial x^{\delta}} \xi-\frac{\rho \xi t^{4 \alpha}}{\Gamma(\alpha+1)^{2} \Gamma(\alpha+4)} \frac{\partial^{\beta}}{\partial x^{\beta}} \xi-\frac{\sigma t^{3 \alpha}}{\Gamma(\alpha+3)} \frac{\partial^{\kappa}}{\partial x^{\kappa}} \xi\right) \\
& -\alpha\left(\frac{\gamma t^{2 \alpha}}{\Gamma(\alpha+2)} \frac{\partial^{\delta}}{\partial x^{\delta}} \xi-\frac{\rho \xi t^{3 \alpha}}{\Gamma(\alpha+1)^{2} \Gamma(\alpha+3)} \frac{\partial^{\beta}}{\partial x^{\beta}} \xi-\frac{\sigma t^{2 \alpha}}{\Gamma(\alpha+2)} \frac{\partial^{\kappa}}{\partial x^{\kappa}} \xi\right) \\
& \times \frac{\partial^{\beta}}{\partial x^{\beta}}\left(\frac{\gamma t^{3 \alpha}}{\Gamma(\alpha+3)} \frac{\partial^{\delta}}{\partial x^{\delta}} \xi-\frac{\rho \xi t^{4 \alpha}}{\Gamma(\alpha+1)^{2} \Gamma(\alpha+4)} \frac{\partial^{\beta}}{\partial x^{\beta}} \xi-\frac{\sigma t^{3 \alpha}}{\Gamma(\alpha+3)} \frac{\partial^{\kappa}}{\partial x^{\kappa}} \xi\right) \\
& -\beta \frac{\partial^{\kappa}}{\partial x^{\kappa}}\left(\frac{\gamma t^{3 \alpha}}{\Gamma(\alpha+3)} \frac{\partial^{\delta}}{\partial x^{\delta}} \xi-\frac{\partial^{4 \alpha}}{\Gamma(\alpha+1)^{2} \Gamma(\alpha+4)} \frac{\partial^{\beta}}{\partial x^{\beta}} \xi-\frac{\sigma t^{3 \alpha}}{\Gamma(\alpha+3)} \frac{\partial^{\kappa}}{\partial x^{\kappa}} \xi\right) .
\end{aligned}
$$

Now, taking the limit as $p \longrightarrow 1$ in equation (20), we get

Next, equations (23)-(28) imply that

$$
u(x, t)=\sum_{i=0}^{\infty} u_{i}(x, t) .
$$

$$
\begin{aligned}
& u(x, t)= g(x)+\left(\gamma \frac{\partial^{\delta}}{\partial x^{\delta}} u_{0}-\rho u_{0} \frac{\partial^{\beta}}{\partial x^{\beta}} u_{0}-\sigma \frac{\partial^{\kappa}}{\partial x^{\kappa}} u_{0}\right) \frac{t^{\alpha}}{\Gamma(\alpha+1)} \\
&+\frac{\gamma t^{2 \alpha}}{\Gamma(\alpha+2)} \frac{\partial^{\delta}}{\partial x^{\delta}} \xi-\frac{\rho \xi t^{3 \alpha}}{\Gamma(\alpha+1)^{2} \Gamma(\alpha+3)} \frac{\partial^{\beta}}{\partial x^{\beta}} \xi-\frac{\sigma t^{2 \alpha}}{\Gamma(\alpha+2)} \frac{\partial^{\kappa}}{\partial x^{\kappa}} \xi \\
&+\gamma \frac{\partial^{\delta}}{\partial x^{\delta}}\left(\frac{\gamma t^{3 \alpha}}{\Gamma(\alpha+3)} \frac{\partial^{\delta}}{\partial x^{\delta}} \xi-\frac{\rho \xi t^{4 \alpha}}{\Gamma(\alpha+1)^{2} \Gamma(\alpha+4)} \frac{\partial^{\beta}}{\partial x^{\beta}} \xi-\frac{\sigma t^{3 \alpha}}{\Gamma(\alpha+3)} \frac{\partial^{\kappa}}{\partial x^{\kappa}} \xi\right) \\
&-\alpha\left(\frac{\gamma t^{2 \alpha}}{\Gamma(\alpha+2)} \frac{\partial^{\delta}}{\partial x^{\delta}} \xi-\frac{\rho \xi t^{3 \alpha}}{\Gamma(\alpha+1)^{2} \Gamma(\alpha+3)} \frac{\partial^{\beta}}{\partial x^{\beta}} \xi-\frac{\sigma t^{2 \alpha}}{\Gamma(\alpha+2)} \frac{\partial^{\kappa}}{\partial x^{\kappa}} \xi\right) \\
& \times \frac{\partial^{\beta}}{\partial x^{\beta}}\left(\frac{\gamma t^{3 \alpha}}{\Gamma(\alpha+3)} \frac{\partial^{\delta}}{\partial x^{\delta}} \xi-\frac{\partial^{\beta}}{\Gamma(\alpha+1)^{2} \Gamma(\alpha+4)} \frac{\sigma t^{3 \alpha}}{\partial x^{\beta}} \xi-\frac{\partial^{\kappa}}{\Gamma(\alpha+3)} \xi\right) \\
&-\beta \frac{\partial^{\kappa}}{\partial x^{\kappa}}\left(\frac{\gamma t^{3 \alpha}}{\Gamma(\alpha+3)} \frac{\partial^{\delta}}{\partial x^{\delta}} \xi-\frac{\rho \xi t^{4 \alpha}}{\Gamma(\alpha+1)^{2} \Gamma(\alpha+4)} \frac{\partial^{\beta}}{\partial x^{\beta}} \xi-\frac{\sigma t^{3 \alpha}}{\Gamma(\alpha+3)} \frac{\partial^{\kappa}}{\partial x^{\kappa}} \xi\right)+\cdots \\
& \Gamma=\gamma \frac{\partial^{\delta}}{\partial x^{\delta}} u_{0}-\rho u_{0} \frac{\partial^{\beta}}{\partial x^{\beta}} u_{0}-\sigma \frac{\partial^{\kappa}}{\partial x^{\kappa}} \xi
\end{aligned}
$$

where 
Hence, (29) is a required solution of the fractional-order KPDE.
4.1. Fractional Temporal Numerical Example. Consider the fractional-order KPDE given by

$$
\frac{\partial^{\alpha} u}{\partial t^{\alpha}}+u \frac{\partial^{\beta} u}{\partial x^{\beta}}+\frac{\partial^{\kappa} u}{\partial x^{\kappa}}-\frac{\partial^{\delta} u}{\partial x^{\delta}}=0,0<\alpha \leq 1,0<\beta \leq 1,2<\kappa \leq 3,4<\delta \leq 5
$$

with initial condition

Using HPM, equation (21) yields that

$$
\begin{gathered}
u(x, 0)=\frac{105}{169} \operatorname{sech}^{4}\left(\frac{x}{2 \sqrt{13}}\right) . \\
H(u, p):=\frac{\partial^{\alpha}}{\partial t^{\alpha}} u-\frac{105}{169} \frac{\partial^{\alpha}}{\partial t^{\alpha}} \operatorname{sech}^{4}\left(\frac{x}{2 \sqrt{13}}\right)+\frac{105}{169} p\left[\frac{\partial^{\alpha}}{\partial t^{\alpha}} \operatorname{sech}^{4}\left(\frac{x}{2 \sqrt{13}}\right)\right] \\
+p\left[u \frac{\partial^{\beta}}{\partial x^{\beta}} u+\frac{\partial^{\kappa}}{\partial x^{\kappa}} u-\frac{\partial^{\delta}}{\partial x^{\delta}} u\right] .
\end{gathered}
$$

Using equations (20) and (33), we get the following comparison with respect to $p$ :

$$
\begin{aligned}
p^{0} & :=\frac{\partial^{\alpha}}{\partial t^{\alpha}} u_{0}-\frac{105}{169} \frac{\partial^{\alpha}}{\partial t^{\alpha}} \operatorname{sech}\left(\frac{x}{2 \sqrt{13}}\right)=0 \\
p^{1}: & =\frac{\partial^{\alpha}}{\partial t^{\alpha}} u_{1}+\left(\frac{105}{169}\right)^{2} \operatorname{sech}\left(\frac{x}{2 \sqrt{13}}\right) \frac{\partial^{\beta}}{\partial x^{\beta}} \operatorname{sech}^{4}\left(\frac{x}{2 \sqrt{13}}\right) . \\
& +\frac{105}{169} \frac{\partial^{\kappa}}{\partial x^{\kappa}} \operatorname{sech}^{4}\left(\frac{x}{2 \sqrt{13}}\right)-\frac{105}{169} \frac{\partial^{\delta}}{\partial x^{\delta}} \operatorname{sech}^{4}\left(\frac{x}{2 \sqrt{13}}\right)=0
\end{aligned}
$$

Zeroth-order problem

From equation (34), we get the zeroth-order problem or $u(x, 0)$ :

$$
u_{0}=\frac{105}{169} \operatorname{sech}^{4}\left(\frac{x}{2 \sqrt{13}}\right) \text {, if } u(x, 0)=\frac{105}{169} \operatorname{sech}^{4}\left(\frac{x}{2 \sqrt{13}}\right) \text {. }
$$




$$
\begin{aligned}
u_{1}(x, t)= & -\frac{22050 \sqrt{13} t^{\alpha}}{371293 \Gamma(\alpha+1)} \operatorname{sech}^{4}\left(\frac{x}{2 \sqrt{13}}\right) \operatorname{sech}\left(\frac{x \sqrt{13}}{26}\right)^{4} \tanh \left(\frac{x \sqrt{13}}{26}\right)+\frac{735 \sqrt{13} t^{\alpha}}{28561 \Gamma(\alpha+1)} \operatorname{sech}\left(\frac{x \sqrt{13}}{26}\right)^{4} \\
& \times \tanh \left(\frac{x \sqrt{13}}{26}\right)\left(1-\tanh \left(\frac{x \sqrt{13}}{26}\right)^{2}\right)-\frac{840 \sqrt{13} t^{\alpha}}{28561 \Gamma(\alpha+1)} \operatorname{sech}\left(\frac{x \sqrt{13}}{26}\right)^{4} \tanh \left(\frac{x \sqrt{13}}{26}\right)^{3} \\
& +\frac{13755 \sqrt{13} t^{\alpha}}{371293 \Gamma(\alpha+1)} \operatorname{sech}\left(\frac{x \sqrt{13}}{26}\right)^{4} \tanh \left(\frac{x \sqrt{13}}{26}\right)^{3}\left(1-\tanh \left(\frac{x \sqrt{13}}{26}\right)^{2}\right)-\frac{3360 \sqrt{13} t^{\alpha}}{371293 \Gamma(\alpha+1)} \operatorname{sech}\left(\frac{x \sqrt{13}}{26}\right)^{4} \\
& \times \tanh \left(\frac{x \sqrt{13}}{26}\right)^{5}-\frac{4935 \sqrt{13} t^{\alpha}}{371293 \Gamma(\alpha+1)} \operatorname{sech}\left(\frac{x \sqrt{13}}{26}\right)^{4} \tanh \left(\frac{x \sqrt{13}}{26}\right)\left(1-\tanh \left(\frac{x \sqrt{13}}{26}\right)^{2}\right)^{2}
\end{aligned}
$$
that

If we use $p \longrightarrow 1$, then solution of equation (31) implies

$$
\begin{aligned}
u(x, t)= & u_{0}+u_{1}(x, t)+u_{2}(x, t)+\cdots u(x, t)=\frac{105}{169} \operatorname{sech}^{4}\left(\frac{x}{2 \sqrt{13}}\right) \\
& -\frac{22050 \sqrt{13} t^{\alpha}}{371293 \Gamma(\alpha+1)} \operatorname{sech}^{4}\left(\frac{x}{2 \sqrt{13}}\right) \operatorname{sech}\left(\frac{x \sqrt{13}}{26}\right)^{4} \tanh \left(\frac{x \sqrt{13}}{26}\right) \\
& +\frac{735 \sqrt{13} t^{\alpha}}{28561 \Gamma(\alpha+1)} \operatorname{sech}\left(\frac{x \sqrt{13}}{26}\right)^{4} \tanh \left(\frac{x \sqrt{13}}{26}\right)\left(1-\tanh \left(\frac{x \sqrt{13}}{26}\right)^{2}\right)-\frac{840 \sqrt{13} t^{\alpha}}{28561 \Gamma(\alpha+1)} \\
& \times \operatorname{sech}\left(\frac{x \sqrt{13}}{26}\right)^{4} \tanh \left(\frac{x \sqrt{13}}{26}\right)^{3}+\frac{13755 \sqrt{13} t^{\alpha}}{371293 \Gamma(\alpha+1)} \operatorname{sech}\left(\frac{x \sqrt{13}}{26}\right)^{4} \tanh \left(\frac{x \sqrt{13}}{26}\right)^{3}\left(1-\tanh \left(\frac{x \sqrt{13}}{26}\right)^{2}\right) \\
& -\frac{3360 \sqrt{13} t^{\alpha}}{371293 \Gamma(\alpha+1)} \operatorname{sech}\left(\frac{x \sqrt{13}}{26}\right)^{4} \tanh \left(\frac{x \sqrt{13}}{26}\right)^{5}-\frac{4935 \sqrt{13} t^{\alpha}}{371293 \Gamma(\alpha+1)} \operatorname{sech}\left(\frac{x \sqrt{13}}{26}\right)^{4} \tanh \left(\frac{x \sqrt{13}}{26}\right) \\
& \times\left(1-\tanh \left(\frac{x \sqrt{13}}{26}\right)^{2}\right)^{2}+\cdots
\end{aligned}
$$

In Figures 1-4, we present graphical presentation of solutions.

\section{Fractional Spatial Numerical Solution}

Consider the equation with orders $0<\beta \leq 1,2<\kappa \leq 3,4$ $<\delta \leq 5$ for fractional spatial numerical solution, that is,

$$
\frac{\partial^{\alpha} u}{\partial t^{\alpha}}+u \frac{\partial^{\beta} u}{\partial x^{\beta}}+\frac{\partial^{\kappa} u}{\partial x^{\kappa}}-\frac{\partial^{\delta} u}{\partial x^{\delta}}=0, \quad 0<\alpha \leq 1
$$

In this equation, for the fractional spatial solution, we only consider the first fractional derivative with order $\beta$ for the sake of eliminating long calculations. Therefore, the firstorder problem is turn out to be as follows.

First-order problem 


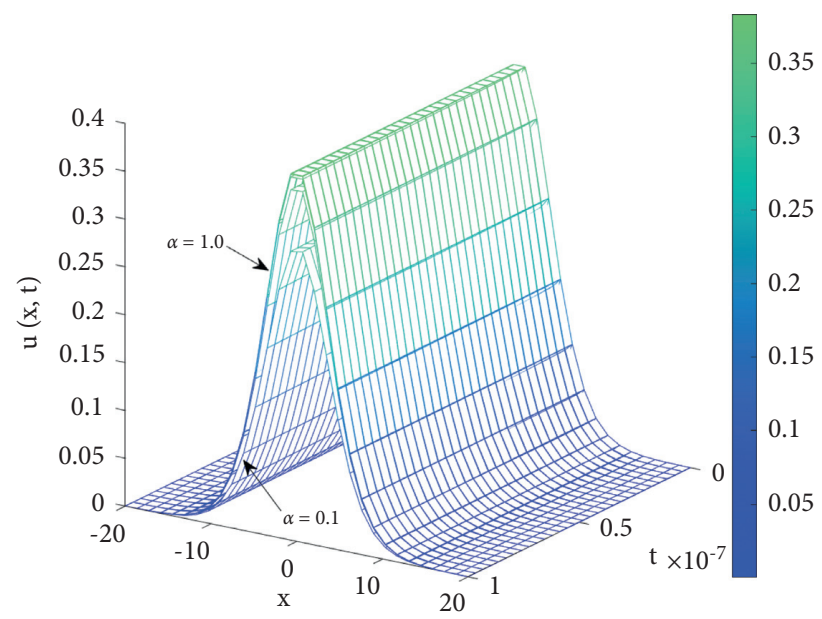

Figure 1: 3D fractional temporal numerical solution $u(x, t)$ for order $\alpha \in(0,1], \kappa=4$, and $\delta=5$.

$$
\begin{aligned}
u_{1}(x, t)= & \frac{11025 t^{\alpha}}{28561 \Gamma(\alpha+1)} \operatorname{sech}^{4}\left(\frac{x}{2 \sqrt{13}}\right) \frac{\partial^{\beta}}{\partial x^{\beta}} \operatorname{sech}^{4}\left(\frac{x}{2 \sqrt{13}}\right)+\frac{105 t^{\alpha}}{169 \Gamma(\alpha+1)} \frac{\partial^{3}}{\partial x^{3}} \operatorname{sech}^{4}\left(\frac{x}{2 \sqrt{13}}\right) \\
& -\frac{105 t^{\alpha}}{169 \Gamma(\alpha+1)} \frac{\partial^{5}}{\partial x^{5}} \operatorname{sech}^{4}\left(\frac{x}{2 \sqrt{13}}\right)=0,
\end{aligned}
$$

implies that

$$
\begin{aligned}
u_{1}(x, t)= & \frac{105 t^{\alpha}}{169 \Gamma(\alpha+1)} \operatorname{sech}^{4}\left(\frac{x}{2 \sqrt{13}}\right)\left(\frac{5.93227042 \times 10^{-10}}{\Gamma(2-\beta)} x^{(1-\beta)}+\frac{0.04779241642}{\Gamma(3-\beta)} x^{(2-\beta)}\right. \\
& \left.+\frac{1.104877850 \times 10^{-6}}{\Gamma(4-\beta)} x^{(3-\beta)}\right)+\frac{735 \sqrt{13} t^{\alpha}}{28561 \Gamma(\alpha+1)} \operatorname{sech}\left(\frac{x \sqrt{13}}{26}\right)^{4} \tanh \left(\frac{x \sqrt{13}}{26}\right) \\
& \times\left(1-\tanh \left(\frac{x \sqrt{13}}{26}\right)^{2}\right)-\frac{840 \sqrt{13} t^{\alpha}}{28561 \Gamma(\alpha+1)} \operatorname{sech}\left(\frac{x \sqrt{13}}{26}\right)^{4} \tanh \left(\frac{x \sqrt{13}}{26}\right)^{3} \\
& +\frac{13755 \sqrt{13} t^{\alpha}}{371293 \Gamma(\alpha+1)} \operatorname{sech}\left(\frac{x \sqrt{13}}{26}\right)^{4} \tanh \left(\frac{x \sqrt{13}}{26}\right)^{3}\left(1-\tanh \left(\frac{x \sqrt{13}}{26}\right)^{2}\right) \\
& -\frac{3360 \sqrt{13} t^{\alpha}}{371293 \Gamma(\alpha+1)} \operatorname{sech}\left(\frac{x \sqrt{13}}{26}\right)^{4} \tanh \left(\frac{x \sqrt{13}}{26}\right)^{5}-\frac{4935 \sqrt{13} t^{\alpha}}{371293 \Gamma(\alpha+1)} \operatorname{sech}\left(\frac{x \sqrt{13}}{26}\right)^{4} \\
& \times \tanh \left(\frac{x \sqrt{13}}{26}\right)\left(1-\tanh \left(\frac{x \sqrt{13}}{26}\right)^{2}\right)^{2} .
\end{aligned}
$$

Hence, the solution at $p \longrightarrow 1$ becomes 

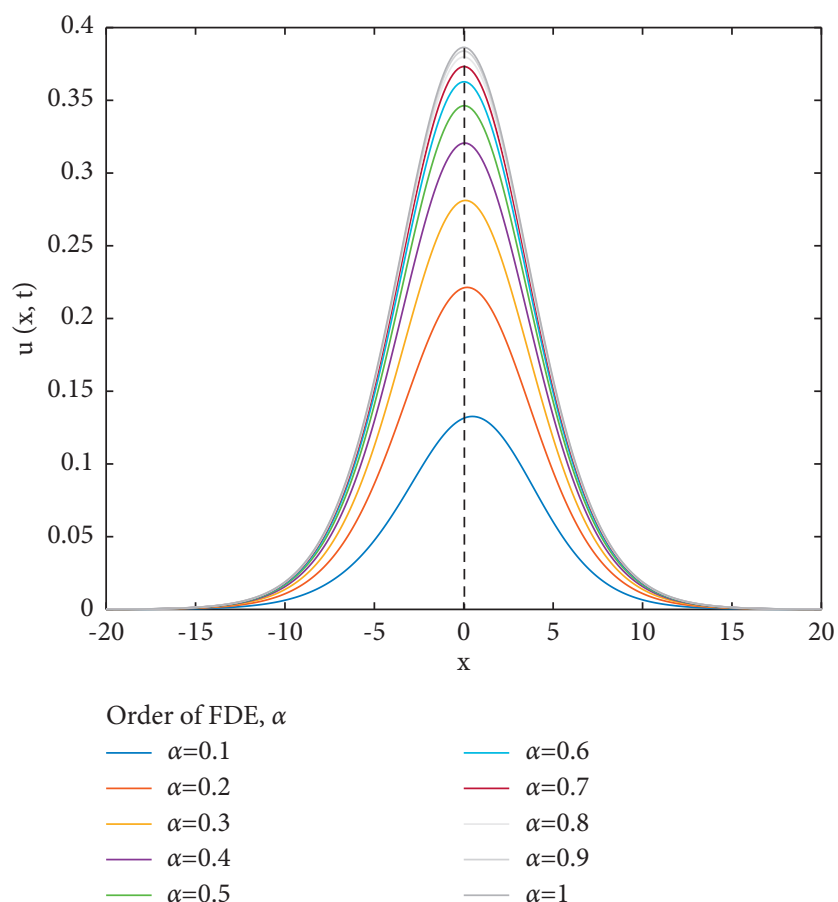

Figure 2: 2D fractional temporal numerical solution $u(x)$ for order $t=0.01, \kappa=4$, and $\delta=5$.

$$
u(x, t)=\sum_{i=0}^{\infty} u_{i}(x, t)
$$

which implies that

$$
\begin{aligned}
u(x, t)= & u_{0}+u_{1}(x, t)+u_{2}(x, t)+\cdots \\
u(x, t)= & \frac{105}{169} \operatorname{sech}^{4}\left(\frac{x}{2 \sqrt{13}}\right)+\frac{105 t^{\alpha}}{169 \Gamma(\alpha+1)} \operatorname{sech}^{4}\left(\frac{x}{2 \sqrt{13}}\right)\left(\frac{5.93227042 \times 10^{-10}}{\Gamma(2-\beta)} x^{(1-\beta)}\right) \\
& +\frac{105 t^{\alpha}}{169 \Gamma(\alpha+1)} \operatorname{sech}^{4}\left(\frac{x}{2 \sqrt{13}}\right)\left(\frac{0.04779241642}{\Gamma(3-\beta)} x^{(2-\beta)}+\frac{1.104877850 \times 10^{-6}}{\Gamma(4-\beta)} x^{(3-\beta)}\right) \\
& +\frac{735 \sqrt{13} t^{\alpha}}{28561 \Gamma(\alpha+1)} \operatorname{sech}\left(\frac{x \sqrt{13}}{26}\right)^{4} \tanh \left(\frac{x \sqrt{13}}{26}\right)\left(1-\tanh \left(\frac{x \sqrt{13}}{26}\right)^{2}\right)-\frac{840 \sqrt{13} t^{\alpha}}{28561 \Gamma(\alpha+1)} \\
& \times \operatorname{sech}\left(\frac{x \sqrt{13}}{26}\right)^{4} \tanh \left(\frac{x \sqrt{13}}{26}\right)^{3}+\frac{13755 \sqrt{13} t^{\alpha}}{371293 \Gamma(\alpha+1)} \operatorname{sech}\left(\frac{x \sqrt{13}}{26}\right)^{4} \tanh \left(\frac{x \sqrt{13}}{26}\right)^{3} \\
& \times\left(1-\tanh \left(\frac{x \sqrt{13}}{26}\right)^{2}\right)-\frac{3360 \sqrt{13} t^{\alpha}}{371293 \Gamma(\alpha+1)} \operatorname{sech}\left(\frac{x \sqrt{13}}{26}\right)^{4} \tanh \left(\frac{x \sqrt{13}}{26}\right)^{5} \\
& -\frac{4935 \sqrt{13} t^{\alpha}}{371293 \Gamma(\alpha+1)} \operatorname{sech}\left(\frac{x \sqrt{13}}{26}\right)^{4} \tanh \left(\frac{x \sqrt{13}}{26}\right)\left(1-\tanh \left(\frac{x \sqrt{13}}{26}\right)^{2}\right)^{2}+\cdots
\end{aligned}
$$

\section{Results and Discussion}

In Figure 1, we have plotted the temporal solution of the fractional-order Kawahara partial differential equation against position $x$ and time $t$ based on equation (35), for different fractional-order $\alpha$, the plot shows that with $\alpha$ amplitude of the solitary wave potential increases while its width squeeze in size slightly. In Figure 2, we have the 


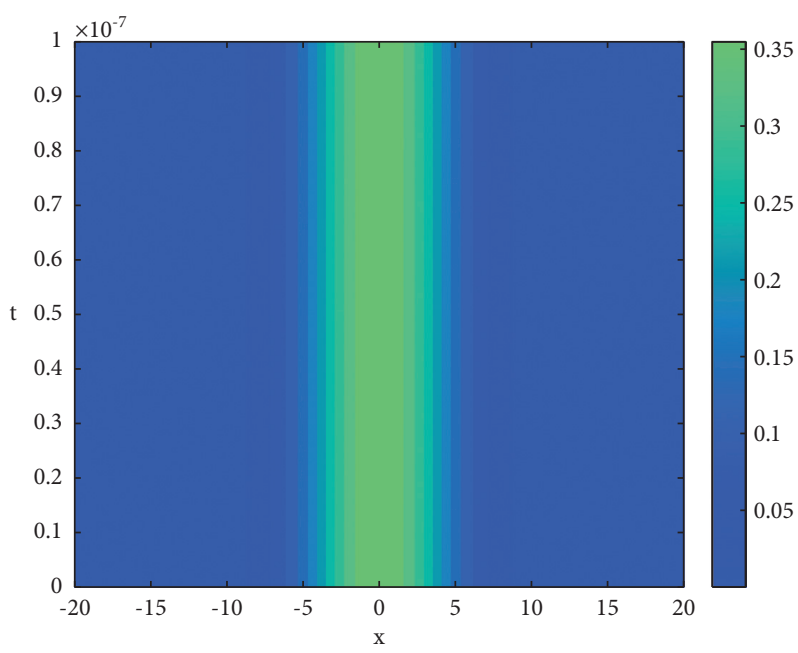

FIgURE 3: Contour of fractional temporal numerical solution $u(x)$ for order $\alpha=0.6, \kappa=4$, and $\delta=5$.

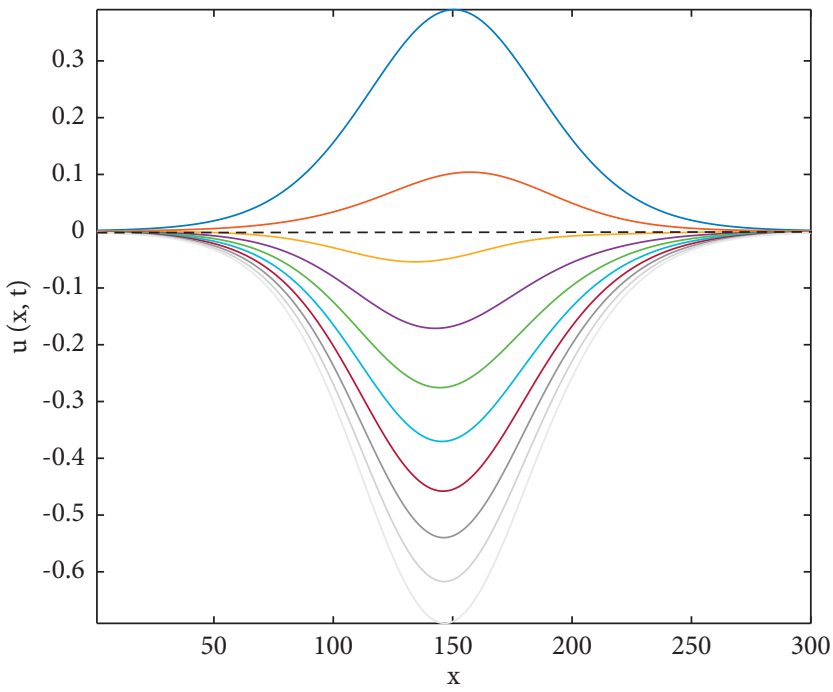

Variations in time, $\mathrm{t}$

$\begin{array}{ll}\mathrm{t}=0 & -\mathrm{t}=2.5 \\ \mathrm{t}=0.5 & -\mathrm{t}=3 \\ \mathrm{t}=1 & -\mathrm{t}=3.5 \\ \mathrm{t}=1.5 & \mathrm{t}=4 \\ \mathrm{t}=2 & \mathrm{t}=4.5\end{array}$

FIGURE 4: 2D fractional temporal numerical solution $u(x)$ for order $\alpha=0.6, \kappa=4$, and $\delta=5$.

comparison of the solitary wave temporal solution $u(x)$ against $x$ for $t=0.01$ and with different fractional-order $\alpha$; this simulation shows a more clear picture of amplitude and dispersion variation with $\alpha$. Figure 3 is the contour plot of solitary wave propagation against $x$ and $t$ for order $\alpha=0.6$. In Figure 4 , the plot is among solitary wave propagation $u(x, t)$ against $x$ and for different time $t$, which shows a very interesting situation of the solitary waves structure; while at the smaller time, we found a compressive type of solitary wave, but when we take the time $t$ greater than 0.6 , then we observe the refractive type of the solitary wave and that waves increase its amplitude with time $t$ and also its dispersion property.

In Figure 5, we have the 3D spatial numerical solution $u(x, t)$ of solitary wave propagation against $x$ and $t$ for differential spatial-order $\beta$ based on equation (40); the simulation shows us that with spatial-order fluctuation, amplitude of the solitary wave change slightly, but the width of the solitary wave change dramatically in greater steps; likewise in Figure 6, we have shown the 2D cross-sectional wave of Figure 5 that demonstrates the amplitude and width of the solitary wave clearly with spatial-order $\beta$ for $t=0.01$. 


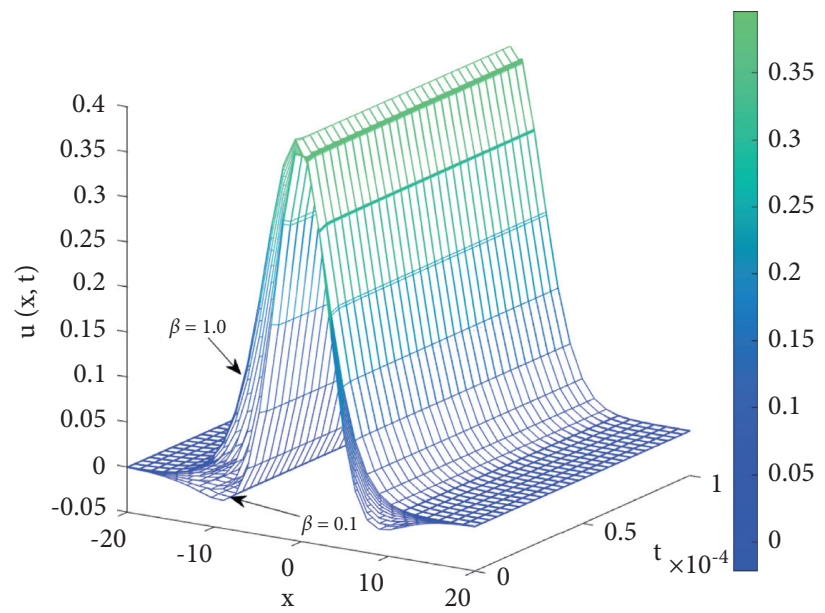

FIGURE 5: 3D fractional spatial numerical solution $u(x, t)$ at order $\beta \in(0,1], \kappa=3, \delta=5$.
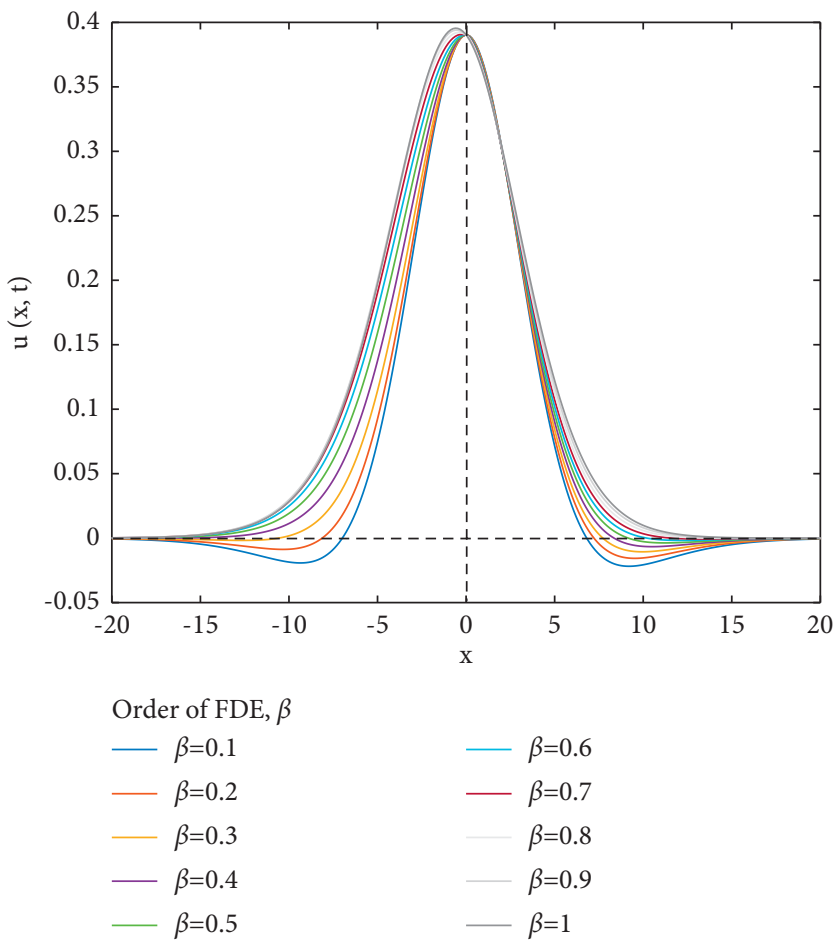

FIgURE 6: 2D fractional spatial numerical solution $u(x)$ at orders $\alpha=0.9, \beta=1, \kappa=3, \delta=5$, and time $t=0.01$. 


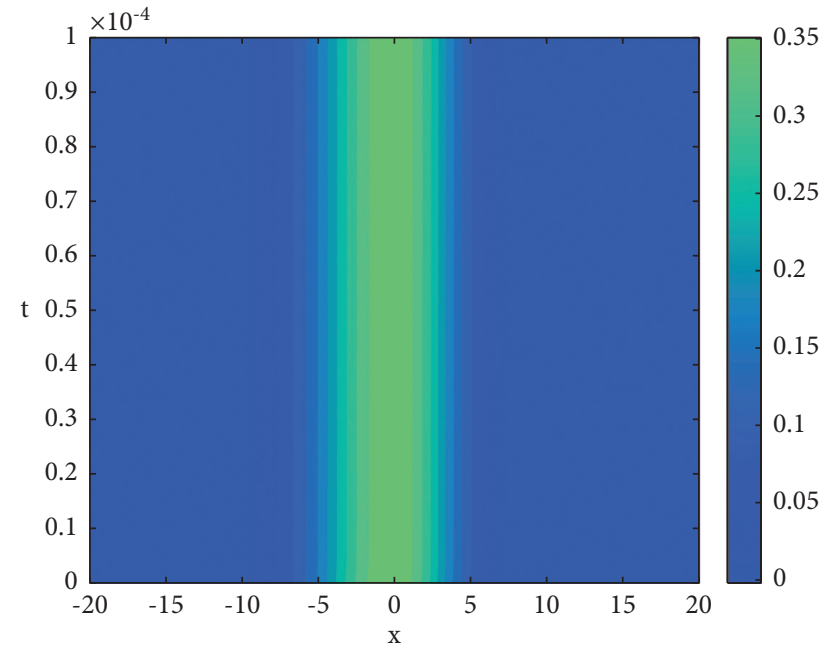

FigUre 7: Contour of fractional spatial numerical solution $u(x, t)$ at orders $\alpha=\beta=0.6, \kappa=3, \delta=5$.

Figure 7 is the contour plot of solitary wave spatial solution against $x$ and $t$.

\section{Conclusion}

Upon the use of the homotopy perturbation method (HPM), we have investigated the Kawahara fractional-order partial differential equation of fifth-order under fractional order. By using Caputo derivative of fractional order separately on temporal and spatial bases, obtained the semianalytical solution for the Kawahara frictional-order differential equation. We have then stimulated various parametric effects (such as $x, t, \alpha$, and $\beta$ ) on the structure of the solitary wave propagation that demonstrates that the width, as well as the amplitude of the solitary wave potential clearly, can change with the change of these parameters. We have shown through our calculation and simulation that He's technique is very useful and power full for the solution of such a higher-order nonlinear partial differential equation. We can extend our calculation to other complex problems especially to the applied side such as astrophysics, plasma physics, and quantum mechanics to solve a complex theoretical calculation by that technique.

\section{Data Availability}

The data used to support the findings of this study are included within the article.

\section{Conflicts of Interest}

The authors declare that they have no conflicts of interest.

\section{Acknowledgments}

The authors would like to acknowledge and express their gratitude to the United Arab Emirates University, Al Ain, UAE, for providing the financial support (\#12S005-UPAR 2020).

\section{References}

[1] D. Kaya and K. Al-Khaled, "A numerical comparison of a Kawahara equation," Physics Letters A, vol. 363, no. 5-6, pp. 433-439, 2007.

[2] A. Dascioglu and S.Ç. Ünal, "New exact solutions for the space-time fractional Kawahara equation," Applied Mathematical Modelling, vol. 89, pp. 952-959, 2021.

[3] T. Özis and I. Aslan, "Application of the $G^{\prime} / G$-expansion method to Kawahara type equations using symbolic computation," Applied Mathematics and Computation, vol. 216, no. 8, pp. 2360-2365, 2010.

[4] K. Muhammet, "Approximate analytic solutions of the modified Kawahara equation with homotopy analysis method," Advances in Difference Equations, vol. 2012, p. 178, 2012.

[5] T. Kawahara, "Oscillatory solitary waves in dispersive media," Journal of the Physical Society of Japan, vol. 33, no. 1, pp. 260-264, 1972.

[6] J.-H. He, "Homotopy perturbation technique," Computer Methods in Applied Mechanics and Engineering, vol. 178, no. 3-4, pp. 257-262, 1999.

[7] J.-H. He, "Comparison of homotopy perturbation method and homotopy analysis method," Applied Mathematics and Computation, vol. 156, no. 2, pp. 527-539, 2004.

[8] M. Sinan, "Analytic approximate solution of Rabies transmission dynamics using Homotopy perturbation method," Matrix Science Mathematic, vol. 4, no. 1, pp. 01-05, 2020.

[9] K. Oldham and J. Spanier, The Fractional Calculus Theory and Applications of Differentiation and Integration to Arbitrary Order, Academic Press, New York, NY, USA, 1974.

[10] K. S. Miller and B. Ross: An Introduction to the Fractional Calculus and Fractional Differential Equations.

[11] I. Podlubny, Fractional Differential Equations, Academic Press, San Diego, CA, USA, 1998.

[12] L. Debnath, "Recent applications of fractional calculus to science and engineering," International Journal of Mathematics and Mathematical Sciences, vol. 2003, pp. 753601-30, 2003.

[13] J. Lu, "Analytical approach to Kawahara equation using variational iteration method and homotopy perturbation method," Topological Methods in Nonlinear Analysis, vol. 31, no. 2, pp. 287-293, 2018.

[14] M. Stefan, "Traveling wave solutions to Kawahara and related equations," Differential Equations and Dynamical Systems, vol. 27, no. 1-3, pp. 19-37, 2019.

[15] A. Ali, M. Y. Khan, M. Sinan et al., "Theoretical and numerical analysis of novel COVID-19 via fractional order mathematical model," Results in Physics, vol. 20, p. 103676, 2021. 